\title{
EDITORIAL
}

\section{Objectivity and subjectivity in forensic psychiatry}

\author{
Elias Abdalla-Filho \\ Department of Ethics and Legal Medicine, Brazilian Psychiatric Association (ABP). Universidade de Brasília (UnB), Brasília, DF, Brazil.
}

Forensic psychiatry is the field of psychiatry that deals specifically with issues of this medical area applied to law. It attempts to clarify, especially to judicial authorities, any doubts that may arise in relation to the mental condition of an individual involved in a legal situation.

The psychiatric report, a document prepared by an expert, tries to provide legal professionals with as much elaborate information as possible. However, accurate and objective responses are not always reached due to the inexact nature of psychiatry and the subjective nature of the object being examined.

As much as one wants to assign an objective character to reports, which is important and necessary, in psychiatric assessment it is not possible to deny the existence of essentially subjective elements, especially if we consider that the subject of study is an individual. Considering that a person can only be considered fully responsible for his/her actions if globally sane, ${ }^{1}$ these elements must necessarily be taken into account. However, subjective elements imply a greater degree of complexity and difficulty in order to be revealed. Disorders specifically related to habits and impulses, as described in the International Classification of Diseases, Tenth Revision (ICD-10), are an example of such conditions, e.g., pathological theft or kleptomania. These disorders have an essentially subjective nature and can keep causal connection with criminal offenses. Then a question arises: how can one objectify the subjective?

It is important to identify when objectivity, perceived by the examiner, is pursued adequately in relation to psychic reality, or when it is overvalued at the expense of subjectivity, creating an unbalanced and therefore mistaken evaluation. There are truly objective aspects, such as a psychotic disorder with diverse manifestations and in causal connection with a bizarre crime, or a history of hospitalizations registered in hospital records. However, the presentation of a mental disorder is not always this obvious. ${ }^{2}$ Unlike this picture, there are other situations that do not present such concrete and objective aspects, but rather a predominance of subjective manifestations, which can be difficult to detect and observe.

When Rogers alerts to the fact that one should not rush in detecting any simulation before some patient's manipulative behavior, ${ }^{3}$ he draws attention to another type of care that the expert must have: not to get carried away by the perception of some very clear objective

Correspondence: Elias Abdalla-Filho, SQN 309, Bloco L, Ap. 603, CEP 70755-120, Brasília, DF, Brazil.

E-mail: elias.abdalla@ terra.com.br element, becoming blind to subjective elements that do not have the same degree of clarity but may also be present. This is especially observed in investigations that are quickly and hastily conducted, and it may also happen in institutions as a result of heavy workload.

When defending the idea of analyzing intraindividual variability by means of risk state exam rather than merely studying interindividual variability through risk status in the examination of violence risk, Douglas \& Skeem are including and valuing the subjective dynamics of the examinee. ${ }^{4}$ In addition to considering the context in which the individual is inserted and other objective elements, they defend the idea of evaluating any changing aspect in risk assessment rather than adopting a static evaluation dictated by the well-known scales widely used in forensic psychiatry.

The examination of an offender's subjective aspects still has a further complicating factor: beyond the subjectivity of certain symptoms of the individual being examined, there is also the expert's own subjectivity. In other words, the subjective manifestations of the offender undergo the expert's personal interpretation. As an example, let us consider an offender's consumption impulse for a given drug. Because of the subjective character of the impulse, one expert may consider it an irresistible impulse, where as another may understand that the impulse could have been fully resisted, but was not. This difference alone would be enough to influence judgment of the determination capacity in relation to the understanding capacity regarding the consumption of the referred drug.

In specific examinations within the criminal sphere in Brazil, the cognitive element is known to be necessary but not sufficient to provide full criminal responsibility. Decision-making for the practice or omission of any act depends on a person's rational capacity, but is not restricted to it; this also includes the emotional universe. Both the unrestrained emotional overdose present in an emotionally unstable personality disorder and the superficiality of typical psychopaths illustrate the influence of emotions on the behavior of offenders.

However, returning to the kleptomania example, the fact that there was an irresistible impulse on the part of the individual does not mean that all theft impulses are necessarily compelling. How can one know whether the impulse felt specifically at the moment of the crime being examined was irresistible or simply not resisted? This question reveals that the dynamism of volition is greater than that of cognition. Also, it shows the high degree of difficulty involved in answering a question always asked 
to experts by legal professionals: the possibility to determine in accordance with the understanding of a matter.

Considering the difficulty of perceived subjectivity, as well as its extreme importance in the completion of a technical report, adequate, thorough training of new professionals is necessary and imperative, especially of those attending residency or other programs aimed at training forensic specialists. At present, unfortunately the opposite is observed: expert unpreparedness. To further aggravate the situation, unprepared professionals are often unaware of their limitations and tend to overvalue objective, cognitive aspects, feel only minimally safe in the work conducted, and produce superficial and misleading conclusions.

\section{Disclosure}

The author reports no conflicts of interest.

\section{References}

1 Meynen G. An ethical framework for assessments of criminal responsibility: applying Susan Wolf's account of sanity to forensic psychiatry. Int J Law Psychiatry. 2012;35:298-304.

2 Abdalla-Filho E, De Souza PA, Tramontina JF, Taborda JG. Mental disorders in prisons. Curr Opin Psychiatry. 2010;23:463-6.

3 Rogers R. Clinical assessment of malingering and deception. 3rd ed. New York: Guilford Press; 2008.

4 Douglas KS, Skeem JL. Violence risk assessment: getting specific about being dynamic. Psychol Public Policy Law. 2005;11:347-83. 